\title{
The effects of lubricant eye drops on visual function as measured by the Inter-blink interval Visual Acuity Decay test
}

This article was published in the following Dove Press journal:

Clinical Ophthalmology

10 September 2009

Number of times this article has been viewed

\section{Gail Torkildsen}

Andover Eye Associates, Andover, MA, USA

Clinicaltrials.gov identifier: NCT00673764

Correspondence: Gail Torkildsen, MD Andover Eye Associates, 138 Haverhill St, Suite 104, Andover, MA 01810, USA

Tel +l 978-475-0705

Email mdlasik@comcast.net
Objective: The purpose of the current study was to evaluate the effects of two marketed ocular lubricants on the visual decay in dry eye patients using the Inter-blink interval Visual Acuity Decay (IVAD) test.

Methods: This controlled, randomized, double-masked crossover study compared the effects of a polyethylene glycol/propylene glycol-based (PEG/PG) tear and a carboxymethylcellulose sodium (CMC)/glycerin tear on the visual acuity decay between blinks of dry eye patients. At visit 1 (Day 0), baseline IVAD measurements were recorded prior to instillation of a single drop of randomized study medication. IVAD testing was repeated at 15-, 45-, and 90-minutes post-instillation. Reading rate and functional blink rate were also evaluated. At the second visit (Day $7 \pm 3$ ), study procedures were repeated using crossover treatment.

Results: Forty-eight (48) subjects with dry eye $(61.1 \pm 14.8$ years old, $79.2 \%$ female, $95.8 \%$ white) completed the study. Treatment with the PEG/PG-based tear demonstrated statistically significantly longer time to one-line loss of best-corrected visual acuity (BCVA) as determined by the IVAD test at 90 minutes post-instillation compared to the $\mathrm{CMC} /$ glycerin tear $(P=0.0365)$. Measurements of median time at BCVA, reading rate, and functional blink rate were similar for both treatments. Both formulations were well tolerated in the population studied.

Conclusions: Treatment with the PEG/PG-based tear demonstrated statistically significant improved maintenance of visual acuity between blinks at 90 minutes post-instillation compared to the $\mathrm{CMC} /$ glycerin tear. This is the first study to demonstrate the ability of an artificial tear to extend visual acuity maintenance between blinks, as measured by the IVAD test.

Keywords: dry eye, visual function, artificial tears, ocular lubricant

\section{Introduction}

The objective signs and subjective symptoms of dry eye fluctuate greatly across patient populations, creating a significant challenge for the clinician to diagnose and treat the condition effectively. A more recent area of study in dry eye is the implications for visual function. The decreased blink rate experienced during visual function tasks, (eg, extended computer use, reading, video gaming, watching TV) can exacerbate dry eye and the signs and symptoms of dry eye (eg, blurred vision, ocular surface staining, short tear film break-up time [TFBUT]), which, in turn, can limit patients' visual functioning capabilities. Not surprisingly, this reciprocal relationship between dry eye and visual tasking can confound the clinician's diagnosis and treatment even further. Patient complaints of the effect of dry eye on visual function may include difficulty driving, reading, and watching television. ${ }^{1}$ 
During each blink of the eye, the action of the upper lid serves the essential purpose of reestablishing the tear film over the ocular surface epithelium. ${ }^{2}$ The tear film instability and increased rates of tear film break-up following a blink that are characteristic of dry eye can be caused by insufficiencies in any of the three major tear film components: lipid, mucin, and aqueous. ${ }^{3,4}$ Ocular surface protection is contingent upon the patient's TFBUT matching or exceeding his or her interblink interval (IBI). ${ }^{5}$ Blink rate is thereby closely integrated with ocular surface integrity.

The effects of dry eye on visual function can be assessed by testing the visual acuity degradation during the IBI. The Inter-blink Interval Visual Acuity Decay (IVAD) test is a novel diagnostic tool that evaluates functional visual acuity between blinks. It is a computer-based visual task which involves identification of Landolt 'c's at individualized bestcorrected visual acuity (BCVA) between blinks and measures parameters based on patient responses. During the test, the rotating optotype ' $c$ ' is presented and the subject responds using a keypad to indicate the direction of the ' $c$ '. ${ }^{1}$ By using a standardized task for all patients before and after treatment, the test is designed to establish an accurate representation of the effects of dry eye treatments on visual function.

A new artificial tear, Systane ${ }^{\circledR}$ Ultra Lubricant Eye Drops (Alcon, Inc., Fort Worth, TX, USA), contains polyethylene glycol 400 (PEG 400) and propylene glycol (PG) as active demulcents, gelling agent HP-Guar, sorbitol, and preservative POLYQUAD. The viscosity is optimized in the bottle with sorbitol to allow for efficient ocular spreading with minimal blur. Upon instillation, the solution then interacts with the mucins and divalent ions of the natural tear film and releases sorbitol, allowing enhanced cross-linking of borate with HP-Guar, and forming a matrix on the ocular surface. ${ }^{6}$ Optive $^{\mathrm{TM}}$ (Allergan, Inc., Irvine, CA, USA) contains carboxymethylcellulose sodium (CMC) and glycerin, and is preserved with Purite. ${ }^{7}$ The drops were chosen as comparators because they contain different polymeric formulations and target similar dry eye populations.

Over-the-counter (OTC) artificial tears are the mainstay in dry eye treatment, and impact the visual function capabilities of patients. Clinical studies of artificial tears have demonstrated differences in blurring effects upon instillation between marketed products, as well as the relationship of residence time to this blurring upon-instillation. ${ }^{89}$ Knowledge of the considerable impact of dry eye on visual function and the implications for quality-of-life led to further investigation of the effects of dry eye treatment on visual function. The present study was designed to evaluate the effect of two lubricant eye drops (Systane Ultra and Optive) on visual function of dry eye patients using the IVAD test.

\section{Methods}

This study utilized a single-center, randomized, double masked, cross-over design to evaluate the visual function effects of two lubricant eye drops in patients with dry eye. The study protocol, protocol amendments, informed consent form, investigator qualifications and site, and recruiting materials were approved by an Institutional Review Board (Southwest Independent; Fort Worth, TX). The study was conducted in accordance with current Good Clinical Practice guidelines and the Declaration of Helsinki.

Subjects were recruited from an existing database of dry eye patients, and were enrolled in the study if they met all inclusion and no exclusion criteria. Enrolled subjects were at least 18 years of age, provided written informed consent, and had a reported history of dry eye in both eyes. Enrolled subjects also had a documented history within the previous 6 months of both a TFBUT $\leq 5$ seconds and a sodium fluorescein corneal staining sum score of $\geq 1$ (on a standardized 0 [none] to 4 [worst] scale ${ }^{10}$ in both eyes. Subjects were enrolled if they reported a use and/or desire to use an artificial tear within the past year, and had a BCVA of $0.6 \log$ MAR or better in each eye using the Early Treatment of Diabetic Retinopathy Study (ETDRS) chart. Subjects were excluded if they had undergone ocular surgery in the previous 6 months, had current punctal occlusion, had a history of intolerance or hypersensitivity to any component of the study medications, or had a history or evidence of external ocular infection. Subjects who had a history or evidence of glaucoma, ocular hypertension, and intraocular inflammation; and those who used systemic medications known to cause ocular drying on an unstable dose for the 30 days prior to visit 1 were also excluded from the study. Subjects who had any ocular or systemic medical condition that might, in the opinion of the investigator, preclude the safe administration of test product, were excluded as well. Use of topical ocular drops within 3 hours of visit 1, use of Restasis $^{\circledR}$ (Allergan) within 30 days of visit 1, and use of either (except in the case of artificial tears) throughout the duration of the study were disallowed.

At visit 1 (day 0) subjects provided written informed consent and signed a Health Insurance Portability and Accountability Act (HIPAA) privacy document prior to any study procedures. Demographic information as well as medical and medication history were obtained from the subject. Each subject's BCVA was tested using the ETDRS chart, 
and primary gaze blink rate was obtained using a digital microcamera and infrared illuminator while the subject was isolated and asked to complete a standard visual task (subjects were not instructed that their blink rate was being monitored). Each subject's functional blink rate was acquired during a practice session of the IVAD test. Reading rate was determined by timing each subject while reading a list of 16 words, one eye at a time. Slit lamp examination of the anterior eye was conducted on both eyes of the subjects.

As subjects qualified, they were assigned enrollment numbers in numerical sequence, and these numbers corresponded to the treatment order for the two test products. After resting for at least 5 minutes, 1 drop of randomized treatment was instilled in both eyes by a designated member of the investigative staff who was not involved in clinical assessment, data management, or data analysis, and who did not disclose treatment assignments to the investigator, sponsor, or patients. Subjects then underwent functional blink rate assessment, IVAD testing, and reading rate evaluation at 15, 45, and 90 minutes post-dose. Finally, any adverse events reported or observed after instillation of test product were recorded and assessed and subjects were scheduled for their next visit. Subjects returned for visit 2 on day $7 \pm 3$, and were queried for artificial tear use in the 3 hours prior to their visit and any adverse event occurrence since visit 1 , and the same method was applied for the cross-over treatment, followed by study exit.

\section{Statistical analysis}

All patients receiving test product were considered evaluable for the safety analysis and all patients receiving test product and completing both visits were considered evaluable for the intent-to-treat (ITT) analysis. All patients who met the criteria for ITT analysis, and satisfied all inclusion and exclusion criteria were considered evaluable for the per-protocol (PP) analysis.

The primary efficacy variables were IVAD test recorded time at BCVA and reading rate of the worse eye as assessed at baseline and 15, 45, and 90 minutes post-dose. Descriptive statistics were presented for each of the efficacy variables (including the mean, standard deviation, sample size, minimum, and maximum) and by treatment and time points. Repeated measures analysis of variance (ANOVA) were used to test for treatment differences in the IVAD test recorded time and reading rate of the worse eye. Descriptive statistics for the secondary variable of functional blink rate assessed at baseline and 15, 45, and 90 minutes post-treatment were generated by treatment and time points. Repeated measures of variance were used to test for treatment differences in functional blink rate. Post hoc exploratory efficacy analysis was conducted using survival analyses (right censored) performed for IVAD time at BCVA at 15, 45, and 90 minutes post-dose. Observations were censored if time at BCVA was equal to IBI.

\section{Results}

Fifty-three subjects were screened and 48 subjects were enrolled in the study, completed all visits, and were evaluable for safety, ITT and PP analyses (mean age $=61.1 \pm 14.8$ years; $20.8 \%$ male; $95.8 \%$ white, $2.1 \%$ black, $2.1 \%$ other). Since the ITT and PP data sets were identical, analyses were performed only on the ITT set.

The results for time at BCVA, reading rate, and functional blink rate measurements are listed in Table 1. A significant difference in survival distribution for time at BCVA (time to one-line loss of BCVA) evaluated using the IVAD test was observed at 90 minutes post-dose: $50 \%$ of patients demonstrated time to one-line loss of BCVA greater than 9.17 seconds in PEG 400/PG treatment group compared to 6.84 seconds in $\mathrm{CMC} /$ glycerin treatment group (Wilcoxon test, $P=0.037$; see Table 2, Figure 1). Repeated measures ANOVA (used to compare treatment differences in mean scores by time because ANOVA allows tests for treatmentby-time and treatment-by-sequence interactions) showed no significant between-treatment differences for time at BCVA overall $(P=0.49)$ or any time point $(P>0.36)$, for reading rate overall $(P=0.082)$ or at any timepoint $(P>0.20)$, or for functional blink rate overall $(P=0.1408)$ or at any timepoint $(P>0.14)$. No adverse events related to the test product were reported and no patients discontinued due to an adverse event.

\section{Discussion}

The current study was designed to demonstrate the visual function impact of two lubricant eye drops through the use of the IVAD test. The IVAD test has been utilized in prior studies, demonstrating the statistically significantly shorter time at which dry eye patients could maintain their BCVAs compared to normals $(P=0.0001) .{ }^{1}$ The present study utilized this clinical tool in order to investigate the potential benefits of artificial tear use on these visual function parameters in dry eye patients.

Although no significant between-treatment differences were observed in the mean values of reading rate or functional blink rate at any time point post-treatment, the survival distribution for time at BCVA demonstrated a statistically 
Table I Efficacy variables

\begin{tabular}{lllll}
\hline & $\begin{array}{l}\text { Time post-treatment } \\
\text { (minutes) }\end{array}$ & $\begin{array}{l}\text { PEG 400/PG/HP-Guar, } \\
\text { mean (SD) }\end{array}$ & \multicolumn{2}{l}{$\begin{array}{l}\text { CMC/glycerin, } \\
\text { mean (SD) }\end{array}$} \\
\hline IVAD time & Baseline & $6.6(4.1)$ & $6.3(4.9)$ & 0.78 \\
at BCVA (sec) & 15 & $7.4(5.4)$ & $8.4(13.1)$ & 0.63 \\
& 45 & $7.4(5.6)$ & $9.4(13.9)$ & 0.36 \\
& 90 & $8.7(9.9)$ & $8.3(15.5)$ & 0.84 \\
Reading & Baseline & $10.9(3.6)$ & $10.7(3.3)$ & 0.55 \\
rate (sec) & 15 & $11.4(4.9)$ & $11.0(3.0)$ & 0.37 \\
& 45 & $11.3(3.8)$ & $10.8(3.0)$ & 0.21 \\
Functional blink & 90 & $10.8(3.3)$ & $10.5(2.5)$ & 0.39 \\
rate (sec) & Baseline & $5.7(5.4)$ & $6.9(5.1)$ & $0.04 *$ \\
& 15 & $5.9(5.5)$ & $5.8(4.7)$ & 0.79 \\
& 45 & $5.9(5.4)$ & $6.7(4.9)$ & 0.17 \\
\hline
\end{tabular}

*Statistically significant at $P \leq 0.05$.

Abbreviations: BCVA, best-corrected visual acuity; IVAD, Inter-blink interval Visual Acuity Decay; PEG, polyethylene glycol; PG, propylene glycol; CMC, carboxymethylcellulose sodium.

significant difference between treatments at 90 minutes post-dose favoring the PEG 400/PG formulation (Wilcoxon test, $P=0.0365)$. The ability of the PEG 400/PG formulation to extend the amount of time to one-line loss of BCVA for prolonged periods of time, as demonstrated by the IVAD test, may suggest beneficial visual effects for patients. The link between visual function loss and diminished quality-of-life is well established in literature. ${ }^{11,12}$ The ability of ocular lubricants to stabilize the tear film during visual tasking and to retard the visual acuity decay between blinks likely translates to enhanced visual functioning capabilities for patients.

Previous research has explored the residence time and duration of action of lubricant eye drops. A prior study compared the duration of tear film stability achieved by the CMC/glycerin tear to that of a PEG 400/PG tear (a formulation with similar active ingredients to that used in the present study). The results of the study demonstrated that the PEG 400/PG drop achieved significantly greater extension of TFBUT at 45 , 60, and 90 minutes post-instillation than the $\mathrm{CMC} /$ glycerin drop $(P<0.05)$. While similar ocular surface protection was determined to result with both artificial tears immediately following instillation, the PEG 400/PG tear was able to prolong TFBUT and maintain positive changes in Ocular Protection Index up to 90 minutes post-instillation. ${ }^{13}$ These results may suggest the prolonged duration of action of the PEG 400/PG formulation used in the current study, as noted in the ability to positively impact the time to one-line loss of BCVA 90 minutes post-instillation. Additionally, previous work has shown that the PEG 400/PG formulation used in this study has enhanced rheological properties, including those resulting from the dilution of sorbitol upon introduction to the tear film, which may also help to explain the effect at 90 minutes. ${ }^{14}$

While this data is important, no significant differences from baseline in BCVA maintenance existed for either drug until 90 minutes post-instillation, when both tears appeared to show their maximum effectiveness. The ability of an artificial tear to promote visual function abilities has the potential to benefit various dry eye patient populations. In order to elaborate upon the extent of these visual effects, future research could examine the impact of visual function improvement

Table 2 Median time to one-line loss of BCVA as measured by the IVAD test

\begin{tabular}{llll}
\hline $\begin{array}{l}\text { Time post-treatment } \\
\text { (minutes) }\end{array}$ & $\begin{array}{l}\text { PEG 400/PG/HP-Guar, } \\
\text { mean (SD) }\end{array}$ & $\begin{array}{l}\text { CMC/glycerin, } \\
\text { mean (SD) }\end{array}$ \\
\hline Baseline & 5.78 & 5.77 & P value \\
15 & 7.75 & 6.42 & 0.37 \\
45 & 6.46 & 5.51 & 0.65 \\
90 & 9.17 & 6.84 & 0.40 \\
\hline
\end{tabular}

Note: "Statistically significant at $P \leq 0.05$.

Abbreviations: BCVA, best-corrected visual acuity; IVAD, inter-blink interval visual acuity decay; PEG, polyethylene glycol; PG, propylene glycol; CMC, carboxymethylcellulose sodium. 


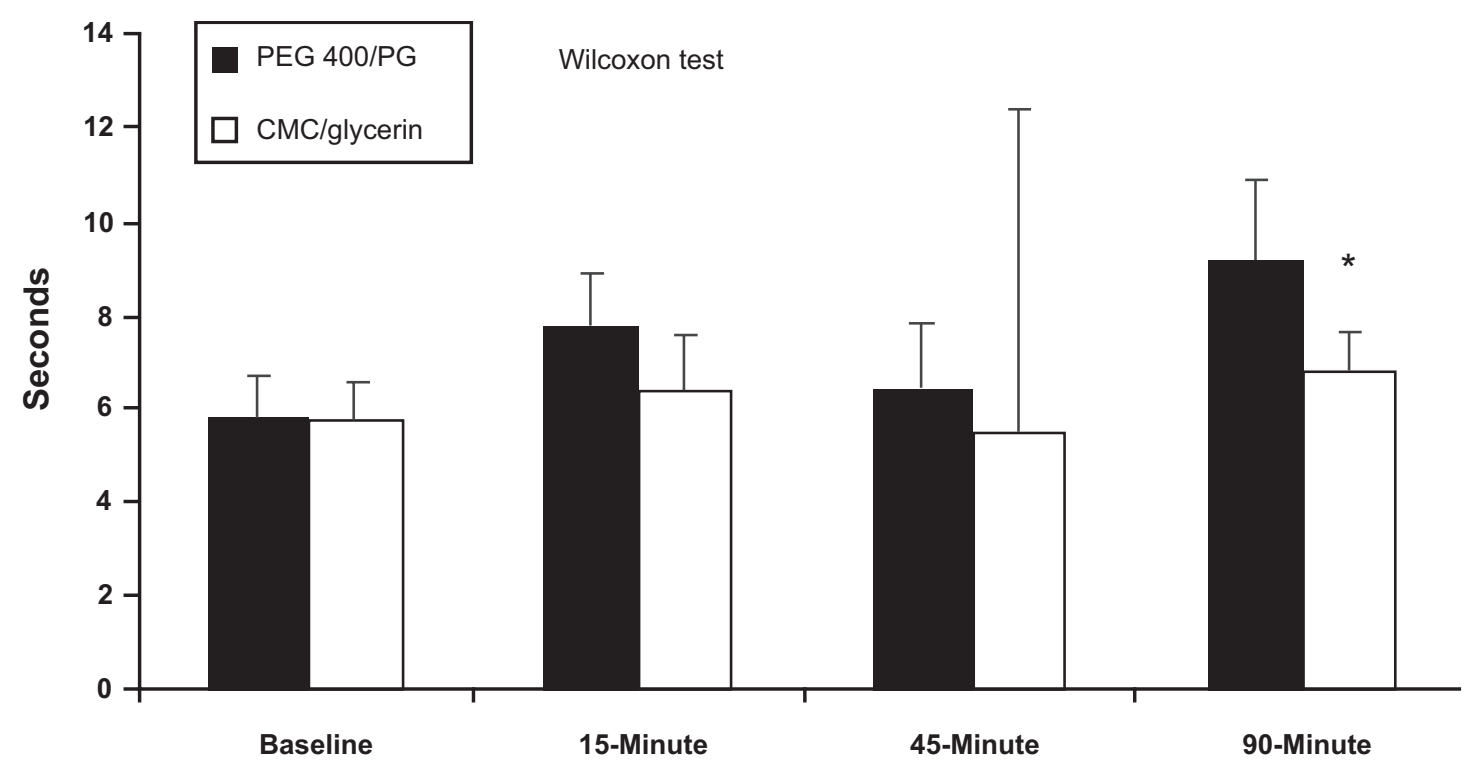

Figure I Median time to one-line loss of best-corrected visual acuity (BCVA) as assessed by the inter-blink interval Visual Acuity Decay (IVAD) test (intent-to-treat population; *P $<0.05$, Wilcoxon test).

Abbreviations: CMC, carboxymethylcellulose sodium; PEG, polyethylene glycol; PG, propylene glycol.

on patient quality-of-life over longer-term artificial tear use; the potential correlation of sign and symptom reduction with visual function improvement; or the use of artificial tears while performing specific visual function tasks (eg, driving at night, computer use, watching television). Investigating the potential effects of artificial tear use on visual function in patients who may not be diagnosed with dry eye, but who may experience ocular discomfort when acutely exacerbated by prolonged periods of visual tasking, could illuminate potential benefits of ocular lubricants in that population as well.

\section{Conclusion}

Both formulations were generally well tolerated in the population studied. No significant between-treatment differences were observed in mean scores for reading rate at any of the time points measured post-treatment. Median time to one-line loss of BCVA as measured using the more sensitive measure of the IVAD test, however, was significantly longer with the PEG 400/PG ocular lubricant than the CMC/glycerin product 90 minutes post-instillation. This is the first clinical trial using the novel IVAD test to demonstrate the ability of lubricant eye drops to extend visual acuity maintenance between blinks.

\section{Disclosures}

This research was supported by Alcon Laboratories, Inc, Fort Worth, TX. Dr Torkildsen has no conflicts of interest regarding this material.

\section{References}

1. Walker P, Ousler GW III, Workman DA, et al. Visual function in normals compared to patients diagnosed with dry eye as measured by the inter-blink interval acuity decay (IVAD) test. Invest Ophthalmol Vis Sci. 2007;48:E-abstract 422.

2. Abelson MB, Doane MG, Ousler G. Tear film and blink dynamics. In: Albert DM, Miller JW, Azar DT, Blodi BA, eds. Principles and Practice of Ophthalmology. Philadelphia: WB Saunders Co; 2008.

3. Abelson MB, Lamberts DW, eds. Dry Eye Update. Princeton, NJ: Excerpta Medica; 1983.

4. Bron AJ. Diagnosis of dry eye. Surv Ophthalmol. 2001;45(Suppl 2): S221-S226.

5. Ousler GW III, Wilcox Hagberg K, Schindelar M, et al. The ocular protection index. Cornea. 2008;27(5):509-513.

6. Ketelson HA, Davis J, Meadows DL. Characterization of a novel polymeric artificial tear delivery system. Invest Ophthalmol Vis Sci. 2008;49:E-Abstract 112.

7. Kaercher T, Buchholz P, Kimmich F. Treatment of patients with keratoconjunctivitis sicca with Optive: results of a multicenter, open-label observational study in Germany. Clin Ophthalmol. 2009;3:33-39.

8. Christensen MT, Martin AE, David RE. A comparison of comfort/ acceptability and blur profile between PEG/PG and CMC/glycerin based artificial tears in both a dry eye and non-dry eye patient populations. Invest Ophthalmol Vis Sci. 2008;49:E-abstract 109.

9. Hall JQ, Paugh JR, Ridder WH III, Nguyen AL. The relationship between residence time (RT) and the visual effect (VE) of artificial tears (AT) in dry eye subjects. Invest Ophthalmol Vis Sci. 2008;49: E-abstract 110.

10. Ousler GW 3rd, Anderson RT, Osborn KE. The effect of senofilcon A contact lenses compared to habitual contact lenses on ocular discomfort during exposure to a controlled adverse environment. Curr Med Res Opin. 2008;24(2):335-341.

11. Miljanović B, Dana R, Sullivan DA, Schaumberg DA. Impact of dry eye syndrome on vision-related quality of life. Am J Ophthalmol. 2007;143(3):409-415.

12. Walker PM, Ousler GW, Lane K, et al. A comparative investigation of spontaneous blink rates across a series of tasks in dry eye and control patients. Invest Ophthalmol Vis Sci. 2008;49:E-abstract 5317. 
13. D'Arienzo P, Ousler GW, Schindelar MS. A comparison of two marketed artificial tears in improvement of tear film stability as measured by tear film break-up time (TFBUT) and ocular protection index (OPI). Poster presentation, Tear Film and Ocular Surface Society Meeting 2007, Taormina, Sicily.
14. Ketelson HA, Davis J, Meadows D. Extensional rheological properties of artificial tear solutions. Invest Ophthalmol Vis Sci. 2009; 50: E-abstract 4642.

\section{Publish your work in this journal}

Clinical Ophthalmology is an international, peer-reviewed journal covering all subspecialties within ophthalmology. Key topics include: Optometry; Visual science; Pharmacology and drug therapy in eye diseases; Basic Sciences; Primary and Secondary eye care; Patient Safety and Quality of Care Improvements. This journal is indexed on
PubMed Central and CAS, and is the official journal of The Society of Clinical Ophthalmology (SCO). The manuscript management system is completely online and includes a very quick and fair peer-review system, which is all easy to use. Visit http://www.dovepress.com/ testimonials.php to read real quotes from published authors. 